\title{
Deindustrialisierung und Wahlverhalten
}

\section{Eine regionale Analyse der Bundestagswahlen 2017}

Der politische Erfolg der Alternative für Deutschland (AfD) bei der Bundestagswahl 2017 lässt sich auch mit negativen Auswirkungen einer schrumpfenden Beschäftigung im verarbeitenden Gewerbe erklären. Dieser politische Effekt einer schrumpfenden Beschäftigung ist in Regionen mit vitaler industrieller Tradition besonders stark. Die durchgeführte Regression bestätigt, dass Regionen, die in den 1930er Jahren durch einen hohen Anteil an Beschäftigten in der verarbeitenden Industrie gekennzeichnet waren und die heute einen starken Rückgang der industriellen Beschäftigung verzeichnen, deutlich höhere Wahlergebnisse für die AfD haben.

Im sächsischen Freital entfielen bei der Bundestagswahl $201735 \%$ der gültigen Zweitstimmen auf die Alternative für Deutschland (AfD). Die junge Partei ging als stärkste politische Kraft aus dieser Wahl hervor, im gesamten Wahlkreis erzielte die AfD ihr bundesweit bestes Ergebnis. In Pirmasens in der Südwestpfalz erreichte die AfD 16\% und damit eines der besten Ergebnisse im Westteil von Deutschland. Beide Orte weisen bei aller Unterschiedlichkeit wirtschaftshistorische Gemeinsamkeiten auf. Bis zum Zweiten Weltkrieg und darüber hinaus prominente Zentren der Industrialisierung in der Stahl- bzw. der Schuhindustrie schrumpfte die Industriebeschäftigung in den letzten 30 Jahren im Zuge der transformations- und globalisierungsbedingten Öffnung der Märkte dramatisch. Waren im vormaligen Kreis Freital Anfang der 1980er Jahre über 25.000 Personen im produzierenden Gewerbe beschäftigt, so waren es 2016 im entsprechenden Gebiet weniger als 8.000 , ein Rückgang von ungefähr $70 \%$. In Pirmasens, das zwar nicht vom Transformationsschock, aber von der ausländischen Importkonkurrenz getroffen wurde, liegt der entsprechende Rückgang bei immerhin $50 \% .^{1}$ Die These dieses Beitrags besagt, dass die Wahlerfolge des neuen politischen Akteurs erstens durch manifeste ökonomische Verluste zumindest mitbedingt sind und zweitens diese Verluste vor allem dann wirksam werden, wenn sie von einem hohen ökonomischen Status aus von den Betroffenen historisch bewertet werden.

(C) Der/die Autor(en) 2020. Open Access: Dieser Artikel wird unter der Creative Commons Namensnennung 4.0 International Lizenz (https:// creativecommons.org/licenses/by/4.0/deed.de) veröffentlicht.

Open Access wird durch die ZBW - Leibniz-Informationszentrum Wirtschaft gefördert.

1 Für den westlichen Teil ließe sich als vermutlich prominentestes Beispiel auch auf Gelsenkirchen verweisen, mit einem AfD-Stimmenanteil von $17 \%$ bei der Bundestagswahl 2017 und einem Beschäftigungsrückgang im produzierenden Gewerbe von $70 \%$ seit den frühen 1980er Jahren.

\section{Ökonomische Disruption, sozialer Status} und Wahlverhalten

Aus theoretischer Sicht steht hinter dieser Hypothese die verhaltensökonomische Erkenntnis, dass Verlusterfahrungen deutlich stärker motivieren als Gewinnerwartungen und dass die Bewertung der Phänomene als Verlust oder Gewinn über Referenzpunkte vermittelt wird (Tversky und Kahneman, 1981). Im vorliegenden Fall sind die Referenzpunkte wirtschaftsgeschichtlich markiert. In Regionen, die durch eine lange Industrietradition gekennzeichnet sind, wirkt derselbe quantitative Verlust, z. B. bei der Beschäftigung, deutlich stärker auf die Identität von Personen als in Gebieten, für die dies nicht zutrifft. Der Wahlerfolg der AfD - so die Hypothese - wäre daher dort am größten, wo eine negative Beschäftigungsentwicklung in der Industrie auf eine lange Industrietradition trifft.

Natürlich ist es wenig plausibel, eine Eruption im Wahlverhalten, wie die der Bundestagswahl 2017, durch langfristige Wirtschaftsentwicklungen erklären zu wollen. Hinter Eruptionen stehen zumeist andere Eruptionen, die als Auslösemechanismen fungieren. Im vorliegenden Fall ist der Migrationsschock der letzten Jahre unbestreitbar ein solcher Impuls. Dieser Impuls hat aber die Regionen Deutschlands durch die Technokratie der Flüchtlingsverteilung in nahezu identischer Weise getroffen. Die politi-

Prof. Dr. Lutz Schneider lehrt Volkswirtschaftslehre an der Hochschule für angewandte Wissenschaften in Coburg und ist Forschungsprofessor am LeibnizInstitut für Wirtschaftsforschung Halle (IWH). 
sche Reaktion war demgegenüber sehr heterogen, und das nicht nur hinsichtlich des Wahlverhaltens bei der Bundestagswahl 2017. Diese regionale heterogene Absorption des Schocks könnte durch in der Vergangenheit erfahrene ökonomische Statusverluste und weitere Abstiegserwartungen bedingt sein.

Dass eine massive De-Industrialisierung traditionsreicher Industriestandorte zu eruptiven Effekten in sozialer und politischer Dimension führen kann, wird schon seit längerer Zeit in den USA beobachtet (Autor et al., 2016; Che et al., 2016; Autor et al., 2019). In Deutschland wurde den Folgen regionaler De-Industrialisierungspozesse für das Wahlverhalten von ökonomischer Seite bisher weniger Beachtung geschenkt - wohl deshalb, weil die Handelsintegration (zumindest in Westdeutschland) per Saldo nicht zu einem Rückgang der Industriebeschäftigung geführt hat (Dauth et al., 2014). Die regionale Variation ist allerdings erheblich und in Ostdeutschland muss von einem flächendeckenden Rückgang historischen AusmaBes gesprochen werden. Eine der wenigen Studien, die den Zusammenhang von Handelsintegration, regionaler Industriebeschäftigung und Wahlverhalten in deutschen Regionen untersucht, stammt von Dippel et al. (2020). Die AfD ist in dieser Studie noch nicht repräsentiert, da sich der Betrachtungszeitraum von 1987 bis 2009 erstreckt. Dennoch liefert die elaborierte Analyse den belastbaren und für den vorliegenden Zusammenhang entscheidenden Befund, dass hohe Importkonkurrenz zu negativen Effekten für die Industriebeschäftigten in den betroffenen Regionen und dieser adverse Beschäftigungseffekt zu signifikanten Stimmengewinnen im rechten Parteienspektrum führt.

Eigens mit dem Wahlerfolg der AfD bei der Bundestagswahl 2017 beschäftigen sich Franz et al. (2018) sowie Rösel und Samartzidis (2018). Die erste Untersuchung betont die Bedeutung ökonomischer und demografischer Faktoren. Demnach punktet die AfD in ländlichen Regionen mit alternder Bevölkerung, aber auch Regionen mit eher unterdurchschnittlichem Einkommen und höherer Industriebeschäftigung neigen der AfD zu. Demgegenüber betonen Rösel und Samartzidis (2018) gesellschaftspolitische Faktoren. Ihrer Untersuchung zufolge erhält die AfD in Regionen besonders viele Stimmen, die durch geringen gesellschaftlichen Zusammenhalt charakterisiert sind, namentlich durch fehlende Diversitätsakzeptanz und geringes Institutionenvertrauen.

\section{Regionale Verteilung: AfD-Hochburgen und Industrietradition}

In Abbildung 1 wird die regionale Verteilung des Zweitstimmenanteils der AfD bei der Bundestagswahl 2017
Abbildung 1

AfD-Zweitstimmenanteil bei der Bundestagswahl 2017

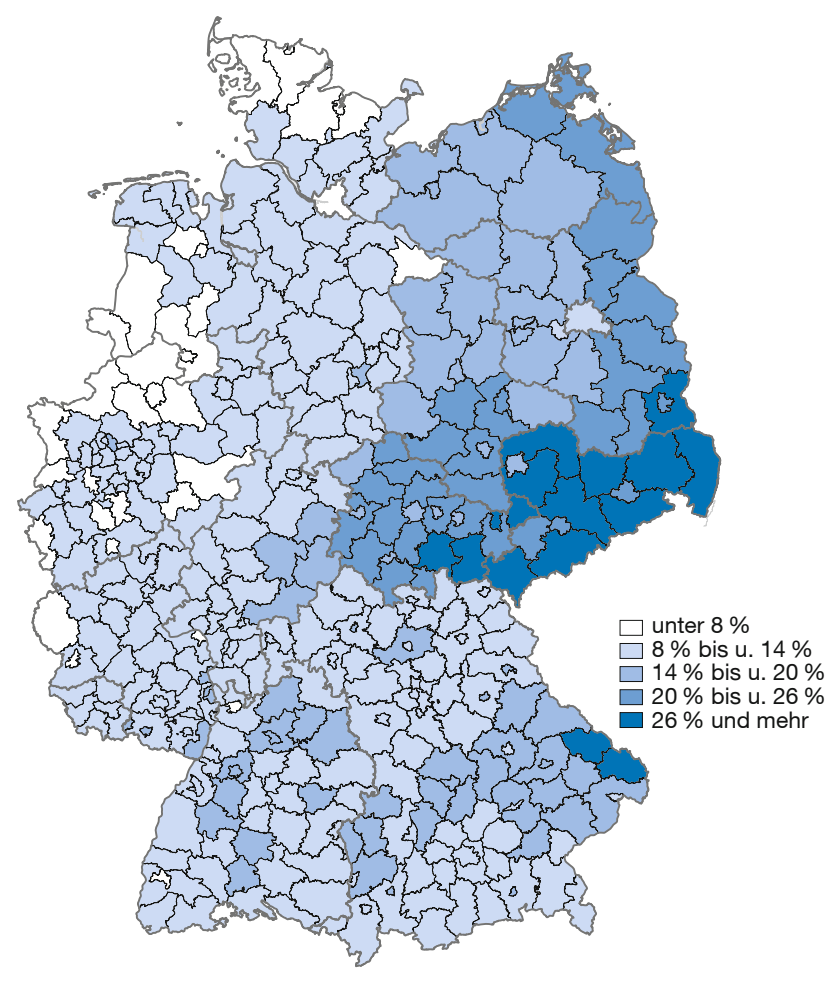

Quelle: Statistisches Bundesamt; eigene Darstellung.

veranschaulicht. Es ist ein klares Ost-West-Gefälle erkennbar. Aggregiert man die fünf neuen Bundesländer, so erreicht die AfD 22,6\% der gültigen Stimmen. In den zehn westlichen Bundesländern kommt sie auf 10,7\%. Allerdings sind unter der generellen Ost-West-Dichotomie auch regionale Variationen zu finden.

Im linken Teil von Abbildung 2 ist die Beschäftigungsentwicklung im produzierenden Gewerbe seit den frühen 1980er Jahren zu sehen. Auch hier findet sich ein deutliches Ost-West-Gefälle. Dieses überlagert allerdings eine starke Heterogenität in den westlichen Bundesländern. Hier gibt es einerseits Regionen mit Verlusten von ostdeutscher Dimension mit einem Beschäftigungsabbau von mehr als $60 \%$. Im Süden und Nordwesten finden sich demgegenüber zahlreiche Regionen mit einem Beschäftigungsaufbau im Produzierenden Sektor.

Der rechte Teil von Abbildung 2 illustriert die historischen Zentren der deutschen Industrie, d. h. Regionen, die in der ersten Hälfte des 20. Jahrhunderts stark durch industrielle Fertigung geprägt gewesen sind. In Kombination der beiden Abbildungen wird deutlich, dass es eine Schnittmenge von Regionen gibt, die in den 1930er Jahren noch hohe Anteile in der Industriebeschäftigung aufwiesen 
Abbildung 2

Beschäftigungsentwicklung im produzierenden Gewerbe und Industriebeschäftigung 1933

Beschäftigungsentwicklung des produzierenden Gewerbes in \% (1980/1981 bis 2016, 401 Kreise)

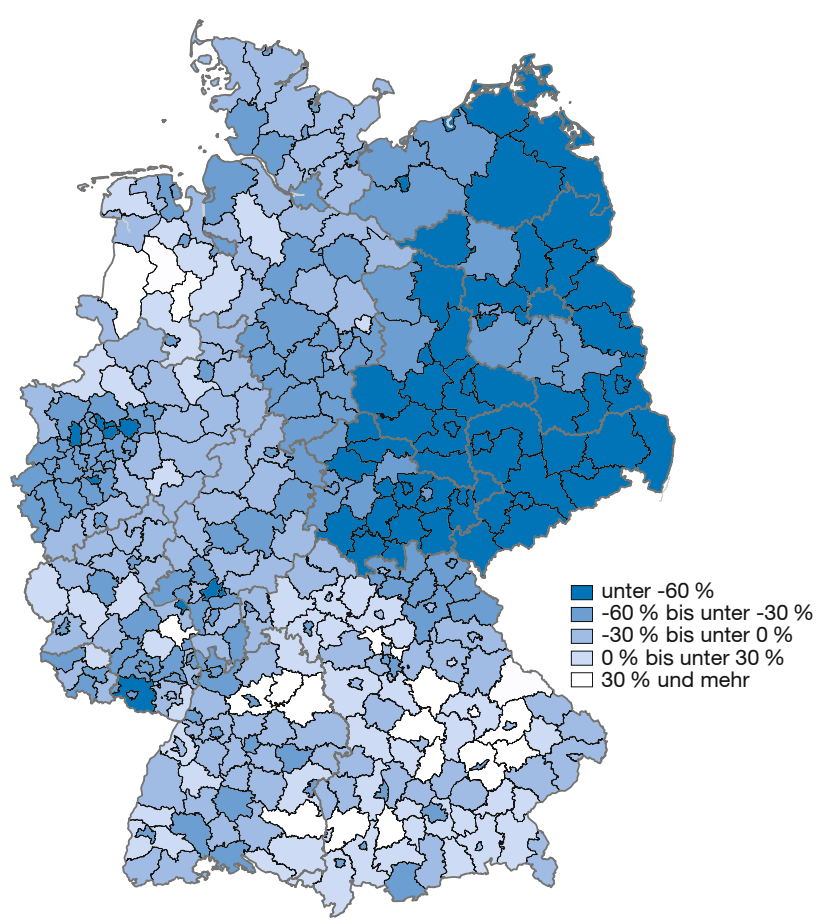

Anteil der Industriebeschäftigten an allen Beschäftigten in \% $(1933,401$ Kreise)

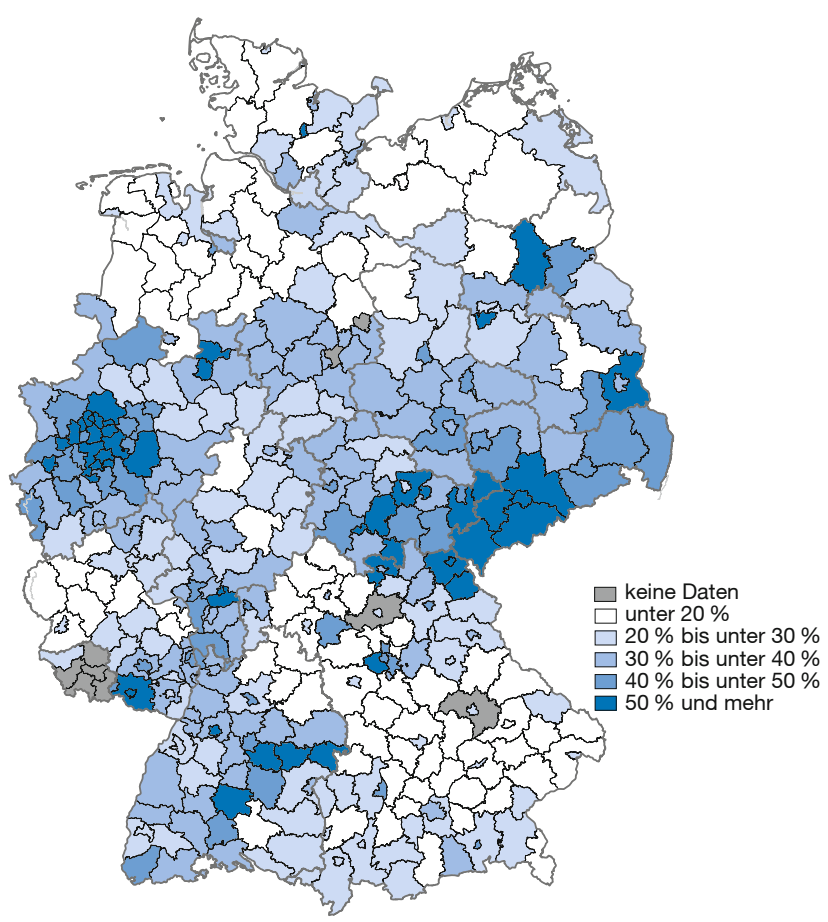

Quellen: Falter, J. W. und D. Hänisch (1990); Statistisches Bundesamt; Beschäftigtenstatistik der Bundesagentur für Arbeit; eigene Darstellung.

(über $40 \%$ ), seit den 1980ern aber zu den Verlierern gehören. Hierzu zählen vornehmlich Sachsen und Thüringen, Teile des Ruhrgebiets und der Pfalz.

Abbildung 3 veranschaulicht den Zusammenhang zwischen dem AfD-Stimmenanteil 2017 und der langfristigen Entwicklung der Industriebeschäftigung getrennt für Regionen mit starker Industrietradition und Regionen ohne eine solche. Ganz generell zeigt sich ein negativer Zusammenhang, der aber klarer und stärker für Regionen mit ausgeprägter Industrietradition erscheint.

\section{AfD stark in traditionellen \\ Industrieregionen ohne Industrie}

In einer multivariaten Analyse werden die regionalen Zuwächse des AfD-Zweitstimmenanteils zwischen 2017 und 2013 - neben anderen Faktoren - durch die Beschäftigungsentwicklung im produzierenden Gewerbe von 1980/1981 bis 2016 erklärt. Dieser Prädiktor wird ferner mit der Information interagiert, ob es sich bei der entsprechenden Region um ein Gebiet mit ausgeprägter Industrietradition handelt. Dazu wird eine Dummy-Variable aufgenommen, die den Wert von eins aufweist, wenn die entsprechende Region im Jahr 1933 beim Anteil der In- dustriebeschäftigten zum oberen Quartil im Deutschen Reich gehörte. Außerdem wird eine Dummy-Variable für die Regionen in den fünf neuen Bundesländern in-

\section{Abbildung 3}

Veränderung der Industriebeschäftigung und AfDZweitstimmenanteil bei der Bundestagswahl 2017

AfD-Stimmenanteil in \%

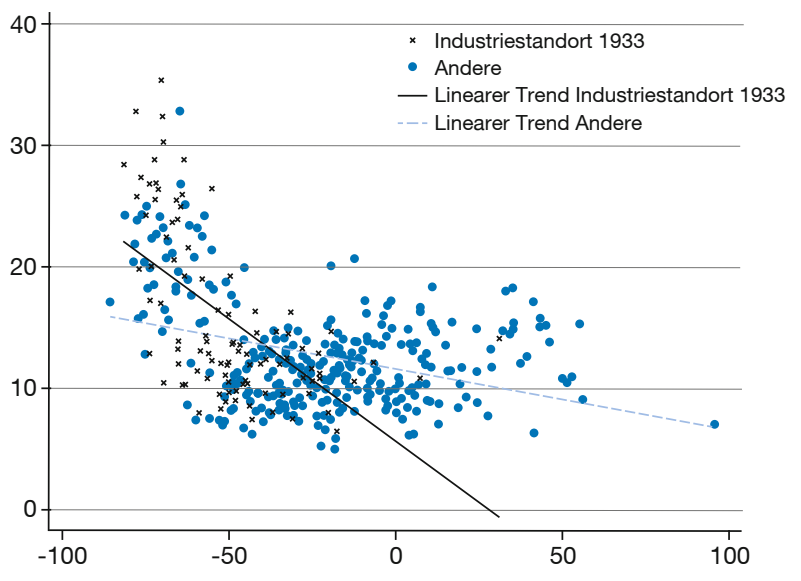

Beschäftigungsveränderung produzierender Sektor 1980/2016 in \% Quelle: eigene Darstellung. 
Tabelle 1

OLS-Schätzung der Veränderung des AfD-Zweitstimmenanteils bei den Bundestagswahlen 2017/2013

\begin{tabular}{|c|c|c|c|c|c|}
\hline \multirow[t]{2}{*}{ Abhängige Variable } & \multicolumn{4}{|c|}{$\begin{array}{l}\text { Veränderung AfD-Zweitstimmenanteil } \\
\qquad 2017 \text { zu } 2013\end{array}$} & $\begin{array}{l}\text { AfD-Zweitstim- } \\
\text { menanteil } 2017\end{array}$ \\
\hline & (1) & (2) & (3) & (4) & (5) \\
\hline Wachstum Beschäftigung 1981/2016 × Industrieschwerpunkt 1933 & $\begin{array}{l}-0,029 \\
{[0,012]^{\star *}}\end{array}$ & $\begin{array}{l}-0,043 \\
{[0,016]^{\star * *}}\end{array}$ & $\begin{array}{l}-0,030 \\
{[0,012]^{\star *}}\end{array}$ & & $\begin{array}{l}-0,038 \\
{[0,013]^{\star * *}}\end{array}$ \\
\hline Industrieschwerpunkt 1933 (0/1-Variable) & $\begin{array}{l}-0,012 \\
{[0,005]^{\star \star}}\end{array}$ & $\begin{array}{l}-0,008 \\
{[0,007]}\end{array}$ & $\begin{array}{l}-0,009 \\
{[0,005]^{\star}}\end{array}$ & & $\begin{array}{l}-0,014 \\
{[0,006]^{\star \star}}\end{array}$ \\
\hline Wachstum Beschäftigung Produzierendes Gewerbe 1981/2016 & $\begin{array}{l}0,022 \\
{[0,006]^{\star * *}}\end{array}$ & $\begin{array}{l}0,013 \\
{[0,006]^{\star *}}\end{array}$ & $\begin{array}{l}0,018 \\
{[0,006]^{\star \star *}}\end{array}$ & & $\begin{array}{l}0,024 \\
{[0,007]^{\star * \star}}\end{array}$ \\
\hline Ostdeutscher Kreis (0/1-Variable) & $\begin{array}{l}0,073 \\
{[0,021]^{\star \star \star}}\end{array}$ & $\begin{array}{l}0,096 \\
{[0,027]^{\star * *}}\end{array}$ & $\begin{array}{l}0,080 \\
{[0,021]^{\star \star \star}}\end{array}$ & & $\begin{array}{l}0,084 \\
{[0,025]^{\star \star \star}}\end{array}$ \\
\hline Wachstum Beschäftigung 1981/2016 × Ostdeutscher Kreis & $\begin{array}{l}-0,054 \\
{[0,030]^{*}}\end{array}$ & $\begin{array}{l}-0,043 \\
{[0,040]}\end{array}$ & $\begin{array}{l}-0,052 \\
{[0,029]^{*}}\end{array}$ & & $\begin{array}{l}-0,062 \\
{[0,036]^{\star}}\end{array}$ \\
\hline Lohnverhältnis Medianlohn Frauen/Medianlohn Männer (2015) & $\begin{array}{l}-0,059 \\
{[0,024]^{\star \star}}\end{array}$ & & $\begin{array}{l}-0,063 \\
{[0,023]^{\star * *}}\end{array}$ & & $\begin{array}{l}-0,038 \\
{[0,028]}\end{array}$ \\
\hline Männeranteil an der Bevölkerung 18 bis 50 Jahre (2015) & $\begin{array}{l}0,512 \\
{[0,122]^{\star \star \star}}\end{array}$ & & $\begin{array}{l}0,498 \\
{[0,120]^{\star \star \star}}\end{array}$ & & $\begin{array}{l}0,486 \\
{[0,135]^{\star \star *}}\end{array}$ \\
\hline Bruttoinlandsprodukt je Einwohner (2015) & $\begin{array}{l}-0,000 \\
{[0,000]^{\star * *}}\end{array}$ & & $\begin{array}{l}-0,000 \\
{[0,000]^{\star * *}}\end{array}$ & & $\begin{array}{l}-0,000 \\
{[0,000]^{\star * *}}\end{array}$ \\
\hline Arbeitslosenquote (2015) & $\begin{array}{l}0,003 \\
{[0,001]^{\star \star \star}}\end{array}$ & & $\begin{array}{l}0,003 \\
{[0,001]^{\star \star *}}\end{array}$ & & $\begin{array}{l}0,003 \\
{[0,001]^{\star \star}}\end{array}$ \\
\hline Bevölkerungsentwicklung (2001 bis 2015) & $\begin{array}{l}-0,165 \\
{[0,031]^{\star \star \star}}\end{array}$ & & $\begin{array}{l}-0,155 \\
{[0,030]^{\star \star \star}}\end{array}$ & & $\begin{array}{l}-0,128 \\
{[0,034]^{\star \star \star}}\end{array}$ \\
\hline Anteil Personen über 60 Jahre (2015) & $\begin{array}{l}-0,092 \\
{[0,071]}\end{array}$ & & $\begin{array}{l}-0,088 \\
{[0,069]}\end{array}$ & & $\begin{array}{l}0,038 \\
{[0,077]}\end{array}$ \\
\hline Anteil Personen mit Migrationshintergrund (2011) & $\begin{array}{l}0,074 \\
{[0,031]^{\star \star}}\end{array}$ & & $\begin{array}{l}0,088 \\
{[0,030]^{\star \star \star}}\end{array}$ & & $\begin{array}{l}0,123 \\
{[0,036]^{\star \star \star}}\end{array}$ \\
\hline Stadtkreis (0/1-Variable) & $\begin{array}{l}-0,007 \\
{[0,004]^{\star}}\end{array}$ & & $\begin{array}{l}-0,009 \\
{[0,004]^{\star \star}}\end{array}$ & & $\begin{array}{l}-0,012 \\
{[0,005]^{\star *}}\end{array}$ \\
\hline SPD-Stimmenanteil Reichstagswahl 1930 & $\begin{array}{l}-0,001 \\
{[0,016]}\end{array}$ & & & $\begin{array}{l}-0,011 \\
{[0,017]}\end{array}$ & $\begin{array}{l}-0,005 \\
{[0,018]}\end{array}$ \\
\hline KPD-Stimmenanteil Reichstagswahl 1930 & $\begin{array}{l}0,049 \\
{[0,025]^{\star \star}}\end{array}$ & & & $\begin{array}{l}0,032 \\
{[0,023]}\end{array}$ & $\begin{array}{l}0,051 \\
{[0,027]^{\star}}\end{array}$ \\
\hline NSDAP-Stimmenanteil Reichstagswahl 1930 & $\begin{array}{l}-0,027 \\
{[0,018]}\end{array}$ & & & $\begin{array}{l}-0,056 \\
{[0,020]^{\star \star \star}}\end{array}$ & $\begin{array}{l}-0,027 \\
{[0,020]}\end{array}$ \\
\hline Zentrum/BVP-Stimmenanteil Reichstagswahl 1930 & $\begin{array}{l}-0,016 \\
{[0,009]^{\star}}\end{array}$ & & & $\begin{array}{l}-0,025 \\
{[0,011]^{\star \star}}\end{array}$ & $\begin{array}{l}-0,027 \\
{[0,010]^{\star *}}\end{array}$ \\
\hline Bestimmtheitsmaß $R^{2}$ & 0,86 & 0,77 & 0,86 & 0,76 & 0,87 \\
\hline Anzahl Kreise & 392 & 392 & 392 & 392 & 392 \\
\hline
\end{tabular}

Anmerkungen: Robuste Standardfehler in Klammern. Die Sterne markieren das Signifikanzniveau: ${ }^{*} 10 \%,{ }^{* *} 5 \%,{ }^{* * *} 1 \%$. In allen Schätzspezifikationen werden fixe Bundeslandeffekte kontrolliert.

Quelle: eigene Berechnungen.

kludiert, auch diese Variable wird zusätzlich mit der Beschäftigtenentwicklung im produzierenden Gewerbe von 1980/1981 bis 2016 interagiert. Damit können offenkundige Ost-West-Unterschiede in der Schätzung berücksichtigt werden. Zudem werden folgende Kontrollvariablen berücksichtigt: das Bruttoinlandsprodukt pro Kopf, die Arbeitslosenquote, die Bevölkerungsentwicklung, der Anteil von Personen über 64, der Anteil von Personen mit Migrationshintergrund, der Männeranteil an der Bevölkerung zwischen 18 und 50 Jahren, das Verhältnis der Frauen- zu den Männerlöhnen sowie die Stimmenanteile der vier erfolgreichsten Parteien bei der Reichstags- wahl im September 1930 (SPD, NSDAP, Zentrum/BVP, KPD). ${ }^{2}$ Außerdem werden Bundesländerspezifika über Bundeslanddummies kontrolliert. Alle Daten wurden auf die regionale Ebene der 401 administrativen Kreise zum Gebietsstand von 2017 transferiert. In Tabelle 1 in Spalte (1) sind die Ergebnisse der Regression der Zuwächse des AfD-Zweitstimmenanteils der Bundestagswahl 2017

2 Die Daten zu den aktuellen Kontrollvariablen aus den Jahren nach 2000 entstammen der Regionalstatistik des Statistischen Bundesamtes bzw. der Beschäftigtenstatistik der Bundesagentur für Arbeit. Die historischen Daten stammen aus Falter und Hänisch (1990). 
gegenüber der Bundestagswahl 2013 auf die genannten Erklärungsgrößen dargestellt.

Der für die vorliegende Arbeit entscheidende Interaktionseffekt ist signifikant negativ. Industrietradition und Beschäftigungsverlust (negatives Beschäftigungswachstum) zusammen sind mit einer höheren Wählergunst für die AfD verbunden. Für sich genommen findet sich in den traditionellen Industrieregionen allerdings eine negative Korrelation zu den AfD-Zuwächsen. In Regionen mit dieser Tradition wählen die Bürger also weniger AfD - es sei denn, in diesen Regionen gab es deutliche Beschäftigungsverluste in der Industrie, mit anderen Worten, die Industrietradition schmilzt ab (Interaktionseffekt). Es zeigt sich ferner eine signifikant positive Korrelation von Beschäftigungsentwicklung im produzierenden Gewerbe und den Stimmengewinnen der AfD bei der Bundestagswahl 2017 im Vergleich zu 2013. Werden die drei Variablen sinnvollerweise zusammen interpretiert, so lässt sich unter Zuhilfenahme der Punktschätzer sagen, dass in Industrieregionen $a b$ einem Rückgang der Industriebeschäftigung von über $41 \%$ die AfD höhere Stimmenzuwächse verzeichnete als in Regionen ohne diese Tradition. Ab dieser Stärke des Beschäftigungsrückgangs macht sich die Industrieidentität im Sinne eines stärkeren Zuwachses an Wählerschaft des neuen politischen Akteurs bemerkbar. Im Osten Deutschlands ist diese Schwelle für alle Industrieregionen erreicht, im Westen für immerhin 42 der 69 Regionen mit starker Industrietradition.

Ferner wird in der Regression sichtbar, dass je höher die Beschäftigungsverluste im Osten sind, desto höher sind auch die Stimmenzuwächse für die AfD. Dieser Effekt ist hoch signifikant und in der Dimension stark; er überkompensiert den generellen positiven Basiseffekt der Beschäftigung im produzierenden Gewerbe deutlich. Ferner zeigt sich ein signifikant positiver Effekt des AfD-Stimmenzuwachses für ostdeutsche Kreise von über 7 Prozentpunkten. Außerdem führt eine geringere Lohnlücke zwischen Männern und Frauen und ein Männerüberschuss in der Altersgruppe der 18- bis 50-Jährigen zu mehr AfD-Stimmen. Darin könnten sich die gesunkene Bedeutung der Männer für die Einkommensgenerierung von Familien sowie die gesunkenen Chancen auf dem Partnerschaftsmarkt widerspiegeln. Zu den schlechteren Beschäftigungs- und Einkommensperspektiven der Männer auf dem Arbeitsmarkt in deindustrialisierten Gebieten gesellen sich Nachteile bei der Partnerwahl (Autor et al., 2019). Auch wenn diese Mechanismen durch die einfache Querschnittsregression nicht identifiziert werden können, so lässt sich aber zumindest behaupten, dass die Korrelationen damit vereinbar sind. Ansonsten zeigt sich, dass ein höheres regionales Einkommen geringere AfDStimmenzuwächse, eine höhere Arbeitslosenquote höhe- re AfD-Stimmengewinne impliziert. In Einklang mit Franz et al. (2018) erreicht die AfD überdies in schrumpfenden Gebieten stärkere Stimmenzuwächse. Ferner zeigt sich eine positive Korrelation von AfD-Stimmengewinnen und dem Anteil von Personen mit Migrationshintergrund. In Stadtkreisen verbucht die AfD geringere Stimmengewinne. Interessanterweise zeigt sich schließlich ein positiver Zusammenhang zwischen den Stimmengewinnen der AfD und den Stimmenanteilen der KPD bei der Reichstagswahl 1930, Regionen mit einem hohen Anteil von Zentrums- und BVP-Wählern 1930 zeigen demgegenüber weniger AfD-Stimmengewinne.

In den Spalten (2) bis (5) wurden andere Schätzspezifikationen verwendet, um die Robustheit der Schätzungen zu prüfen. In Spezifikation (2) wird auf ökonomische und demografische Kontrollvariablen und die historischen Variablen zur Reichstagswahl 1930 gänzlich verzichtet. Es zeigt sich hier sogar ein noch stärkerer Effekt des Zusammenspiels von Industrietradition und Entwicklung der Industriebeschäftigung. In Spezifikation (3), welche die ökonomischen und demografischen Effekte kontrolliert, zeigen sich nur noch geringfügige Unterschiede zur vollen Spezifikation in Spalte (1).

In Spalte (4) wird noch einmal gesondert der Frage von historischen Pfadabhängigkeiten der Wahlergebnisse nachgegangen. In der eindrucksvollen Untersuchung von Cantoni et al. (2019) wird eine positive regionale Korrelation zwischen dem Wahlergebnis der NSDAP in den Reichstagswahlen der Weimarer Republik und den AfDStimmenzuwächsen zwischen 2013 und 2017 belegt. In der vorliegenden Schätzspezifikation (1) zeigte sich hingegen eine positive Korrelation der AfD-Zuwächse zu den KPD-Ergebnissen in der Wahl von 1930. Dies muss freilich nicht im Widerspruch zu Cantoni et al. (2019) stehen, da diese sich ausschließlich den NSDAP-Ergebnissen widmen. Dabei zeigt sich allerdings ein deutlicher Unterschied zu den vorliegenden Ergebnissen. Der von Cantoni et al. (2019) gefundene positive NSDAP-Effekt lässt sich in Spalte (1) nicht auffinden, in Spezifikation (4) - wenn auf andere exogene Variablen gänzlich verzichtet wird ergibt sich in der vorliegenden Analyse sogar eine signifikant negative Korrelation zwischen dem Ergebnis der NSDAP 1930 und den AfD-Stimmenzuwächsen zwischen 2013 und 2017. Dieser erstaunliche Unterschied könnte darin begründet sein, dass Cantoni et al. (2019) eine kleinräumigere regionale Analyseeinheit oder aber eine andere Methode der Zuordnung der historischen Wahldaten auf die heutige Gebietsstruktur verwenden. Dennoch scheint es hier einen Bedarf für weitere Analysen zu geben - auch im Hinblick auf die Frage nach der Korrelation der AfDStimmengewinne zu anderen, nicht rechtsgerichteten Parteien des Weimarer Reichstags. 
In Spalte (5) ist ferner zu erkennen, dass sich die Ergebnisse kaum verändern, wenn statt der Veränderung der Stimmenanteile der AfD zwischen 2013 und 2017 in einer Level-Regression lediglich das AfD-Wahlergebnis 2017 als abhängige Variable verwendet wird. Dies könnte dafür sprechen, dass die Partei 2013 noch eine deutlich andere Wählerschaft angezogen hat, als dies 2017 der Fall war. Dadurch sind die Ergebnisse der Level- und der Differenzen-Schätzung sehr ähnlich.

\section{Fazit}

Der Beitrag liefert Belege für die These, dass die Wahlerfolge der AfD bei der Bundestagswahl 2017 auch mit den regionalen Beschäftigungsrückgängen in der Industrie seit den 1980er Jahren erklärt werden können - zumindest dann, wenn die Beschäftigungsrückgänge hoch (und damit irreversibel) und an traditionsreichen Industriestandorten erfolgt sind. Die präsentierte Querschnittsanalyse gibt in der Tat deutliche Hinweise für einen derartigen Zusammenhang. In weiteren Untersuchungen zeigt sich ferner, dass die Zusammenhänge auch für die Europawahl 2019 belegbar sind, sie gelten im Übrigen nicht für illegitime gewalttätige Formen politischen Protests, z. B. in Form von Übergriffen auf Flüchtlinge.

Aus theoretischer Sicht zeigt die Analyse einmal mehr, dass ökonomische Verwerfungen wirkmächtige Folgen für politische Einstellungen und politisches Verhalten haben. Dabei erhärtet die empirische Untersuchung die verhaltensökonomische Einsicht, dass die subjektiven Bewertungen solcher ökonomischen Verwerfungen entscheidend vom historischen Referenzstandard geprägt werden. Eine ökonomische Schrumpfung wird sich anders auswirken, wenn der Verlust von einem hohen Status aus erfolgt. Und der Verlust wird stärker wirken, wenn dadurch die individuelle und/oder auch die regionale Identität bedroht wird - eine Einsicht der Identitätsökonomik (Akerlof und Cranton, 2000). Hierdurch gewinnt die Quantität der Schrumpfung eine wichtige Bedeutung. Ab einer gewissen kritischen Größenordnung kann eine Krise individuell wie regional als identitätsbedrohend erfahren werden. Die politischen Reaktionen werden in diesem Falle sehr viel einschneidender ausfallen.
Zuletzt muss einschränkend darauf verwiesen werden, dass hinter den in der empirischen Analyse beobachteten Faktoren andere Mechanismen wirksam sein können, die dann auch eine andere Interpretation der Wahlursachen nahelegen würden. Zumindest ein schwer zu übersehendes Identifikationsproblem sei genannt. Die Ergebnisse werden maßgeblich durch die Ost-WestDifferenz geprägt; ist im Osten doch der Beschäftigungsrückgang seit den 1980er Jahren besonders hoch gewesen, ebenso waren es die Stimmenanteile der AfD 2017. Hinter der resultierenden Korrelation könnten sich andere Mechanismen verbergen - abweichende kulturelle und soziale Prägungen, politische Einstellungen und Erwartungshaltungen. Auch wenn der Einbezug der regionalen Dummy-Variablen dieses Problem abmildern dürfte, kann ein verzerrender Effekt nicht vollständig ausgeschlossen werden.

\section{Literatur}

Akerlof, G. A., und R. E. Kranton (2000), Economics and Identity, Quarterly Journal of Economics, 115(3), 715-753.

Autor, D., D. Dorn und G. Hanson (2019), When Work Disappears: Manufacturing Decline and the Falling Marriage Market Value of Young Men, American Economic Review: Insights, 1(2).

Autor, D., D. Dorn, G. Hanson und K. Majlesi (2016), Importing political polarization? The electoral consequences of rising trade exposure, National Bureau of Economic Research, Nr. w22637.

Cantoni, D., F. Hagemeister und M. Westcott (2019), Persistence and Activation of Right-Wing Political Ideology, Rationality and Competition, Discussion Paper Series, Nr. 142.

Che, Y., Y. Lu, J. R. Pierce, P. K. Schott und Z. Tao (2016), Does trade liberalization with China influence US elections?, National Bureau of Economic Research, Nr. w22178.

Dauth, W., S. Findeisen und J. Südekum (2014), The rise of the East and the Far East: German labor markets and trade integration, Journal of the European Economic Association, 12(6), 1643-1675.

Dippel, C., R. Gold, S. Heblich und R. Pinto (2020), The Effect of Trade on Workers and Voters, im Erscheinen.

Falter, J. W. und D. Hänisch (1990), Wahl- und Sozialdaten der Kreise und Gemeinden des Deutschen Reiches von 1920 bis 1933, GESIS Datenarchiv, Köln, ZA8013 Datenfile Version 1.0.0, doi:10.4232/1.8013.

Franz, C., M. Fratzscher und A. S. Kritikos (2018), AfD in dünn besiedelten Räumen mit Überalterungsproblemen stärker, DIW-Wochenbericht, 85(8), 135-144.

Rösel, F. und L. Samartzidis (2018), Wert- statt Geldmangel: AfD triumphiert im Osten dort, wo es an Toleranz und Vertrauen in die Politik fehlt, ifo Dresden berichtet, 25(3), 9-13.

Tversky, A. und D. Kahneman (1981), The framing of decisions and the psychology of choice, Science, 211(4481), 453-458.

Title: Deindustrialisation and Voting Behaviour: A Regional Analysis of the German Federal Election of 2017

Abstract: In this contribution, the political success of the Alternative für Deutschland (AfD) in the German federal election of 2017 is explained by the adverse effect of shrinking manufacturing employment. The employment effect is particularly strong in regions with vital industrial traditions. The performed regression confirms that regions that were characterised by a high share of manufacturing workers in the 1930s and that experience a sharp decline in industrial employment today show significantly better election results for the AfD.

JEL Classification: D72, F6, J2 